\title{
Validation of an image registration and segmentation method to measure stent graft motion on ECG- gated CT using a physical dynamic stent graft model
}

Maaike A. Koenrades, Ella M. Struijs, Almar Klein, Henny Kuipers, Robert H. Geelkerken, et al.

Maaike A. Koenrades, Ella M. Struijs, Almar Klein, Henny Kuipers, Robert H. Geelkerken, Cornelis H. Slump, "Validation of an image registration and segmentation method to measure stent graft motion on ECG-gated CT using a physical dynamic stent graft model," Proc. SPIE 10134, Medical Imaging 2017: Computer-Aided Diagnosis, 1013418 (3 March 2017); doi: 10.1117/12.2254262 


\title{
Validation of an image registration and segmentation method to measure stent graft motion on ECG-gated CT using a physical dynamic stent graft model
}

\author{
Maaike A. Koenrades ${ }^{\mathrm{a}, \mathrm{b}}$, Ella M. Struijs ${ }^{\mathrm{a}}$, Almar Klein ${ }^{\mathrm{a}}$, Henny Kuipers ${ }^{\mathrm{a}}$, \\ Robert H. Geelkerken ${ }^{\mathrm{a}, \mathrm{b}}$, and Cornelis H. Slump ${ }^{\mathrm{a}}$ \\ ${ }^{a}$ MIRA Institute for Biomedical Engineering and Technical Medicine \\ University of Twente, PO Box 217, 7500 AE Enschede, the Netherlands \\ ${ }^{\mathrm{b}}$ Division of Vascular Surgery, Department of Surgery, Medisch Spectrum Twente \\ PO Box 50000, 7500 KA Enschede, the Netherlands
}

\begin{abstract}
The application of endovascular aortic aneurysm repair has expanded over the last decade. However, the longterm performance of stent grafts, in particular durable fixation and sealing to the aortic wall, remains the main concern of this treatment. The sealing and fixation are challenged at every heartbeat due to downward and radial pulsatile forces. Yet knowledge on cardiac-induced dynamics of implanted stent grafts is sparse, as it is not measured in routine clinical follow-up. Such knowledge is particularly relevant to perform fatigue tests, to predict failure in the individual patient and to improve stent graft designs. Using a physical dynamic stent graft model in an anthropomorphic phantom, we have evaluated the performance of our previously proposed segmentation and registration algorithm to detect periodic motion of stent grafts on ECG-gated $(3 \mathrm{D}+\mathrm{t}) \mathrm{CT}$ data. Abdominal aortic motion profiles were simulated in two series of Gaussian based patterns with different amplitudes and frequencies. Experiments were performed on a 64-slice CT scanner with a helical scan protocol and retrospective gating. Motion patterns as estimated by our algorithm were compared to motion patterns obtained from optical camera recordings of the physical stent graft model in motion. Absolute errors of the patterns' amplitude were smaller than $0.28 \mathrm{~mm}$. Even the motion pattern with an amplitude of $0.23 \mathrm{~mm}$ was measured, although the amplitude of motion was overestimated by the algorithm with $43 \%$. We conclude that the algorithm performs well for measurement of stent graft motion in the $\mathrm{mm}$ and sub-mm range. This ultimately is expected to aid in patient-specific risk assessment and improving stent graft designs.
\end{abstract}

Keywords: stent graft motion, experimental validation, image registration, segmentation, ECG-gated CT

\section{INTRODUCTION}

Endovascular aortic aneurysm repair (EVAR) has become widely accepted for the treatment of aortic aneurysm due to its less invasive nature compared to conventional open surgery. In addition, the application of EVAR is rapidly expanding for challenging vascular anatomy using fenestrated and branched stent grafts, chimney grafts and sac-anchoring endoprostheses. ${ }^{1}$ Consequently, EVAR techniques are becoming increasingly complex and involve placement of up to four stents in the renal and mesenteric arteries to preserve perfusion. Durable fixation and sealing of the stent graft to the aortic wall remain the main concern of EVAR. If the sealing of the stent graft fails, then blood flow can enter the aneurysm sac and the risk of rupture returns. Proximal migration and endoleak are responsible for reintervention within 4 years after endovascular treatment in 5-24\% of the patients. ${ }^{1-6}$ Also, there remains the small but not negligible risk of rupture of $1-2 \%$ per year. ${ }^{7}$ The seal and fixation are continuously challenged due to cardiac-induced forces. Every heart beat, the blood flow induces motion of the aorta and the stent graft. This may cause the stent graft to detach from the vessel wall leading to migration, endoleak, and/or kinking. Also, stent fracture may occur due to the continuous cyclic deformation

Further author information: (Send correspondence to M.A.K.)

M.A.K.: E-mail: m.a.koenrades@utwente.nl, Telephone: +31534895135

C.H.S.: E-mail: c.h.slump@utwente.nl, Telephone: +31534892094

Medical Imaging 2017: Computer-Aided Diagnosis, edited by Samuel G. Armato III, Nicholas A. Petrick, Proc. of SPIE Vol. 10134, 1013418 - @ 2017 SPIE · CCC code: 1605-7422/17/\$18 · doi: 10.1117/12.2254262 
that exerts mechanical stress on the wire frame. Depending on the type of stent graft and the location of the fracture, this may have serious consequences. ${ }^{5,8}$ The long-term performance of new and modified stent grafts is difficult to assess, since long-term results of these devices are not yet available. For every new design or modification, stent graft manufacturers use computational modeling and accelerated fatigue testing to optimize their designs and prevent failure in patients. However, these techniques rely on the applied boundary conditions, which are based on assumptions rather than actual knowledge of in vivo cardiac-induced motion. Clinical failure may present long after the first implantation when many patients have already been treated. Detailed knowledge on the dynamics of implanted stent grafts is limited, since routine follow-up imaging is commonly static computed tomography (CT) and does not allow for measurement of motion. Moreover, it is not straightforward to measure motion of single stents inside a patient. ECG-gated $(3 \mathrm{D}+\mathrm{t}) \mathrm{CT}$ can be used to quantify motions of stent grafts $^{9-12}$ and the aortic vessel. ${ }^{13-21}$ Several clinical observational studies have evaluated pulsatile distention of the aorta during the cardiac cycle by measuring aortic dimensions perpendicular to a central luminal line in multiplanar reconstructions at predetermined levels. ${ }^{13-19,22-25}$ A disadvantage of using $2 \mathrm{D}$ cross sectional images is that it does not allow for correction of movement out of plane in cranial or caudal direction. Also, measurements were performed in each of the reconstructed phases, resulting in measurement inconsistencies and relatively high observer variability in the range of the measured motion. We aim to provide a method to quantitatively measure the dynamics of single stent graft components and their interaction with native vessels in vivo in multiphase 3D volumes without having to perform measurements in each of the reconstructed phases by using image registration techniques. Previously, we have proposed the use of a combined registration and segmentation algorithm to quantify stent graft motion in CT data ${ }^{10}$ and found the Gravity registration algorithm to be most suitable for this purpose. ${ }^{26}$ This paper continues this line of work and is the first to evaluate the performance of the combined algorithm on ECG-gated CT.

\section{METHODS}

In real-world ECG-gated CT data, motion during the cardiac cycle is not known. In this paper, we have used a motion simulator to perform a series of validation experiments with a moving stent graft.

\subsection{Experimental setup}

A motion simulator capable of moving in a predetermined pattern with synchronized ECG-triggering (PC controlled phantom device, QRM Quality Assurance in Radiology and Medicine GmbH, Möhrendorf, Germany ${ }^{27}$ ) was used to obtain a series of ECG-gated CT scans with a stent graft moving with different amplitudes at different virtual heart rates. An anthropomorphic aortic abdomen phantom was developed by QRM to mimic a cross section of the human abdomen, comprising of spine, kidneys and soft tissue equivalent material with attenuation values of the human abdomen (dimensions $x y z: 300 \times 200 \times 250 \mathrm{~mm}$; hole diam.: $45 \mathrm{~mm}$ ). An Anaconda stent graft body (Vascutek, Inchinnan, Scotland; body diam. $28 \mathrm{~mm}$, nitinol wire) was attached to the lever of the motion simulator and placed in the center of the abdomen phantom. The stent graft was surrounded by a water filled polyacrylic container with cylindrical tube (outer diam. $45 \mathrm{~mm}$, wall thickness $2 \mathrm{~mm}$ ). The proximal dual ring of the stent graft was fixated in a compressed (oversized) position of approximately $5 \%$ with respect to the manufactured diameter, such that the rings formed a 'saddle' similar to the situation in vivo (Figure 4). The setup with CT scanner is shown in Figure 3.

\subsubsection{Abdominal aortic motion patterns}

A motion pattern composed of a series of Gaussian functions was designed to simulate aortic motion based on given parameters:

$$
\begin{gathered}
P(t)=A \times\left(G\left(t-t_{\text {top }}\right)_{\sigma 1} \times H\left(t_{\text {top }}-t\right)+G\left(t-t_{\text {top }}\right)_{\sigma 2} \times H\left(t-t_{\text {top }}\right)+A_{\text {extra }} \times G\left(t-t_{\text {top_extra }}\right)_{\sigma_{\text {extra }}}\right) \\
G_{\sigma}(t)=e^{-t^{2} / 2 \sigma^{2}} \\
H(t)=\left\{\begin{array}{l}
1, t>0 \\
0, t \leq 0
\end{array}\right\}
\end{gathered}
$$

where $P(t)$ is the position over time $(0 \leq t \leq T), A$ is the amplitude of the pattern, $G_{\sigma}(t)$ and $H(t)$ the Gaussian and Heaviside functions (Equation 2,3) respectively, $\sigma 1$ and $\sigma 2$ the sigma's of the respective Gaussian 
Table 1. Motion patterns of the simulator. Input parameters (Equation 1) are given with the actual executed heart rate and amplitude of the pattern as obtained by camera analysis (ie Output). Mean and standard deviation of the recorded periods are given. The average output periods were used as a reference in the experiments. Note that A3 and B3 are the same signal. The frequency is given as heart rate in beats per minute (bpm).

\begin{tabular}{|c|c|c|c|c|c|c|c|}
\hline \multirow[t]{3}{*}{ Motion pattern } & \multicolumn{2}{|c|}{ Heart rate (bpm) } & \multicolumn{2}{|c|}{ Amplitude (mm) } & \multicolumn{3}{|c|}{ Second peak } \\
\hline & & & & & $A_{\text {extra }}, t_{\text {top_extra }}, \sigma_{\text {extra }}$ & Ampl & ude $(\mathrm{mm})$ \\
\hline & Input & Output & Input & Output & Input & Input & Output \\
\hline $\mathrm{A} 1$ & 50 & $49.0 \pm 0.6$ & 1.0 & $0.72 \pm 0.01$ & - & & - \\
\hline A2 & 100 & $96.3 \pm 2.4$ & 1.3 & $0.75 \pm 0.01$ & - & & - \\
\hline A3 & 75 & $73.3 \pm 1.5$ & 1.2 & $0.70 \pm 0.01$ & - & & - \\
\hline B0 & 75 & $75 \pm 0$ & 0 & $0.02 \pm 0.01$ & - & & \\
\hline B1 & 75 & $74.2 \pm 3.5$ & 0.6 & $0.23 \pm 0.02$ & - & & - \\
\hline B2 & 75 & $73.2 \pm 2.2$ & 0.8 & $0.37 \pm 0.02$ & - & & - \\
\hline B3 & 75 & $73.3 \pm 1.5$ & 1.2 & $0.70 \pm 0.01$ & - & & - \\
\hline B4 & 75 & $73.2 \pm 2.4$ & 2.0 & $1.36 \pm 0.05$ & - & & - \\
\hline B5 & 75 & $73.2 \pm 1.72$ & 2.0 & $1.26 \pm 0.04$ & $0.7,0.8,0.05$ & 1.84 & $1.12 \pm 0.09$ \\
\hline B6 & 75 & $73.1 \pm 1.7$ & 2.0 & $1.19 \pm 0.01$ & $0.7,0.8,0.09$ & 1.84 & $1.19 \pm 0.01$ \\
\hline
\end{tabular}

function, and $t_{t o p}$ the temporal location of the peak. Both sigma's and peak locations are expressed as a fraction of the period T. A second peak can be created by providing $A_{\text {extra }}, t_{\text {top_extra }}$ and $\sigma_{\text {extra }}$.
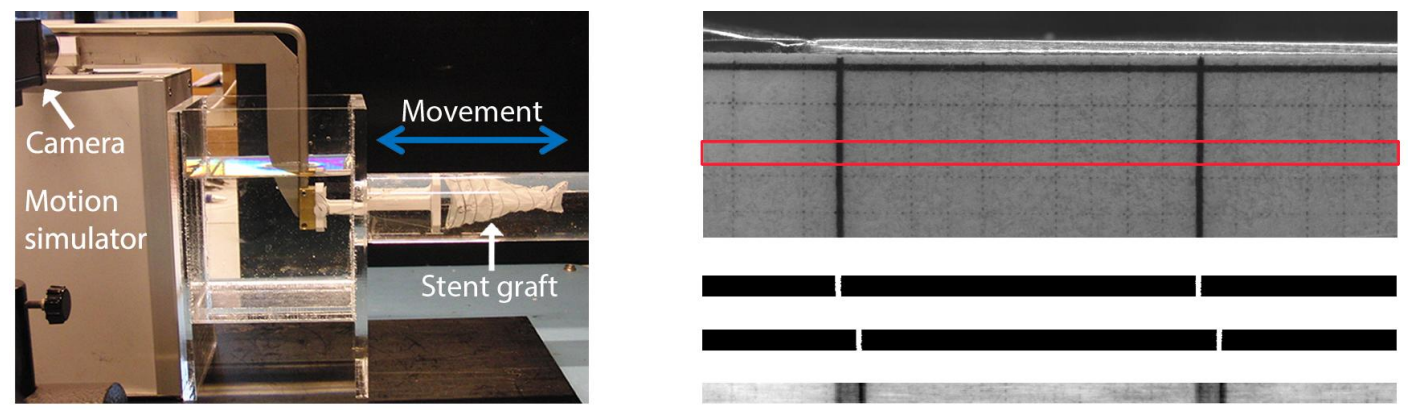

Figure 1. The experimental setup with camera. A camera recorded a mm-grid on the moving motion simulator (left). Grayscale image frames were analyzed (right) by selecting a section of the grid (red rectangle) and applying a binary threshold operation. The result is shown for two frames at the start and end of a period (middle), together with the average of all the image frames that were recorded for this motion pattern (bottom). The displacement patterns (Figure 2) were used as a reference in the experiments.

Two series of motion patterns were generated using Equation 1, with $\sigma 1$ and $\sigma 2$ of $0.4 \times t_{\text {top }}$ and $0.4 \times\left(1-t_{\text {top }}\right)$, respectively and a temporal location of the peak at $35 \%$ of the period, which represents the peak systole in the abdominal aorta. ${ }^{28}$ In the A series the amplitude was constant while the heart rate changed and in the B series vice versa. In the pattern B0 the stent graft did not move. This pattern was included as a control. Amplitudes were chosen relatively small since the amplitude of motion expected in the abdominal aorta is in the order of $1 \mathrm{~mm} \cdot{ }^{13,14,23,29-31}$ Two motion patterns were given a second peak to simulate more complex aortic motion with nonuniform patterns, as was reported by Flora et al. who studied micromovements of the aorta with photogrammetry during open surgery. ${ }^{29}$ Two generated motion patterns are presented in Figure 2 (input simulator). With amplitude, we refer to the maximum absolute value of displacement. The height of the vertical lines in Figure 2 represents the amplitude of the pattern. These lines also indicate the start and end of the period. 

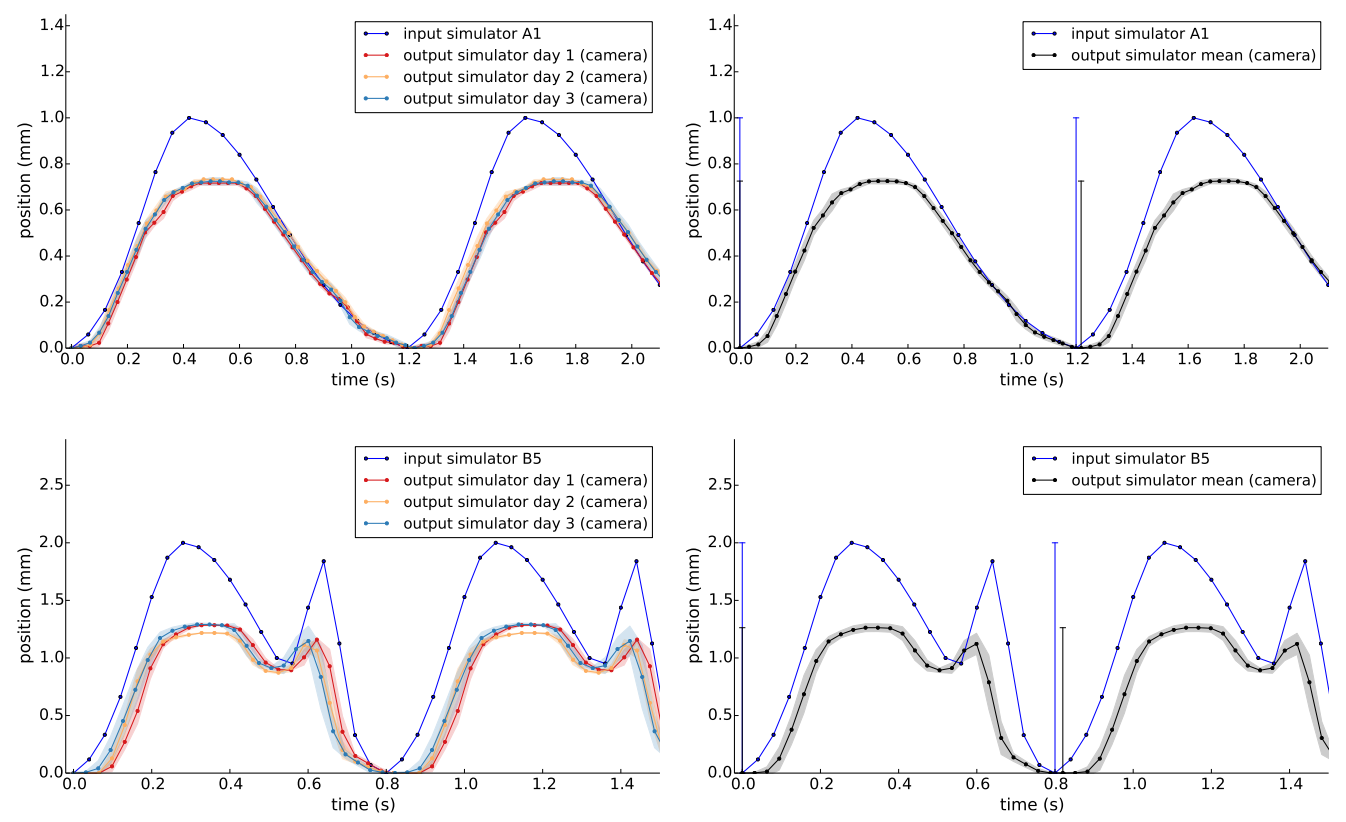

Figure 2. Example of two motion patterns (A1 and B5 in Table 1) that were generated with Equation 1. 'Input simulator' refers to the patterns that were programmed in the motion simulator. The actual executed output patterns that were obtained by analysis of optical camera recordings are referred to as 'Output simulator'. The colored bounds represent the standard deviation around the mean (solid lines) of the recorded periods. The mean output period of the three camera recordings is used as a reference in the experiments (solid black lines on the right).

\subsubsection{Accuracy and precision of the motion simulator}

The motion simulator provides $1 \mathrm{D}$ motions according to preset motion patterns with a maximum amplitude of 50 $\mathrm{mm}$. To evaluate the accuracy of our algorithm to measure stent motion on ECG-gated CT, it is of importance that the motion executed by the simulator is known and precise. Technical specifications of the simulator state that the accuracy of a sinusoidal motion at 140 beats per minute (bpm) is better than $6.3 \%$ and the accuracy in static mode (constant displacement) better than $0.2 \mathrm{~mm}$. However, for motion with small amplitudes the executed motion deviated from to intended motion. We therefore analyzed the executed motion by optical camera recordings of the experimental setup (Figure 1). A Sony XCD-X710 high resolution monochrome camera (30 frames/s; XGA: $1024 \times 768$ pixels) recorded the displacement of an attached mm-grid. Each pattern was recorded for approximately eight periods at three different days. The displacement of $\mathrm{mm}$-grid lines in the images was analyzed in Matlab (2014b) by converting the images to grayscale and applying a threshold. This process in illustrated in Figure 1. Single periods where obtained from the displacement patterns by detecting the local minima. To obtain an average period and the variation over all recorded periods, the single periods were superimposed by time shifting while minimizing the mean squared error. Analysis of the recorded image frames showed that although the executed motion deviated from the preset motion, it was precise and reproducible. Figure 2 shows two preset input patterns and the analyzed output with colored bounds representing the standard deviation. Table 1 presents the input and output values of the heart rate and amplitude for each motion pattern. The mean output periods were used as a reference in the experiments. To perform experiments in $x$-direction, the simulator can be rotated 90 degrees with respect to the lever that is positioned in $z$-direction. The executed motion in $x$-direction was not continuous for the small motion patterns of interest. Experiments were therefore performed in $z$-direction only.

\subsubsection{ECG-gated (3D+t) CT scans}

Experiments were performed on a 64-slice CT scanner (Aquilion, Toshiba Medical Systems Corporation, Tokyo, Japan) with a helical scan protocol that is currently being used in a clinical observational study to the motion 
of the Anaconda stent graft. The experimental setup is shown in Figure 3. ECG-gated scans were obtained by triggering the CT scanner with the motion simulator. Scan and reconstruction parameters were as follows: rotation time, 0.4 seconds; collimation, $64 \times 0.5 \mathrm{~mm}$; slice thickness, $1 \mathrm{~mm}$; increment, $0.5 \mathrm{~mm}$; tube voltage, 120 $\mathrm{kV}$; tube current time product, $60 \mathrm{mAs}$. The pitch factor was set automatically based on the heart rate. Data was reconstructed with the FC12 reconstruction filter, a matrix size of $512 \times 512$, and field of view of approximately $250 \times 250 \mathrm{~mm}$, which resulted in approximately isotropic voxels of $0.5 \mathrm{~mm}^{3}$. Retrospective gating was applied to obtain 10 equidistant volumes covering the cardiac cycle (ie, $0-90 \%$ of the RR-interval). The resulting data were cropped to approximately $200 \times 256 \times 256$ voxels to reduce memory requirements. The setup was scanned while executing the aortic motion patterns given in Table 1.
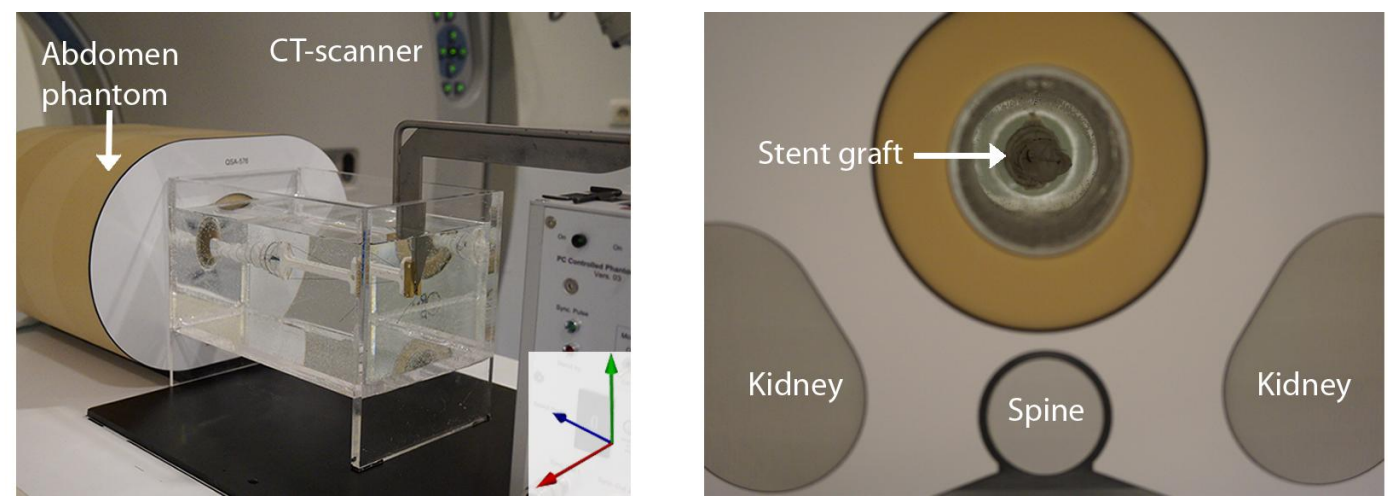

Figure 3. The experimental setup with CT scanner. The stent graft is moving in $z$-direction (left) inside the aortic abdomen phantom (right). The axes of the coordinate system indicate the $x$ (red), $y$ (green) and $z$-direction (blue).

\subsection{Algorithm validation}

Stent graft motion during the cardiac cycle was quantitatively assessed by applying a previously proposed registration and segmentation algorithm. ${ }^{10,32}$ Deformation fields between the reconstructed phases of the ECGgated CT scans were estimated and applied to a geometric model of the stent graft. The deformation fields describe the displacement of each voxel in the volume with respect to the average of all phases. The method involves three steps:

1. Group-wise registration of the reconstructed 3D image phases, resulting in a deformation (ie, vector) field for each phase and a multiphase time-averaged 3D image with noise reduction that is corrected for deformation between phases, hereafter referred to as time-averaged volume.

2. Segmentation of the stent graft in the time-averaged volume, resulting in a geometric model that represents the wire of the stent graft.

3. Applying the deformation fields to the geometric model to quantitatively and qualitatively assess motion of the model.

The registration algorithm combines a B-spline grid for regularization with an image "force" that drives the registration from individually weighted voxels. It was first proposed by Klein et al. ${ }^{33}$ and is available in the Python Image Registration Toolkit (PIRT). ${ }^{34}$ The Gravity algorithm was used to register the reconstructed phases. This algorithm is based on the idea of masses in the images that attract one another. A Laplacian operator was applied for the calculation of the mass images in order to base the attraction of mass on the wire of the stent graft. ${ }^{26}$ Geometric models of the dual ring-stent were obtained from the time-averaged volumes by applying the segmentation algorithm described by Klein et al. ${ }^{32}$ The segmentation algorithm uses a Minimum Cost Path (MCP) method to connect a set of seed points on the wire frame of the stent graft, resulting in geometric model in the form of a graph consisting of nodes and edges. The edges represent the wire frame and the nodes are placed at wire crossings. A subvoxel estimation of the locations has been implemented using 1D 

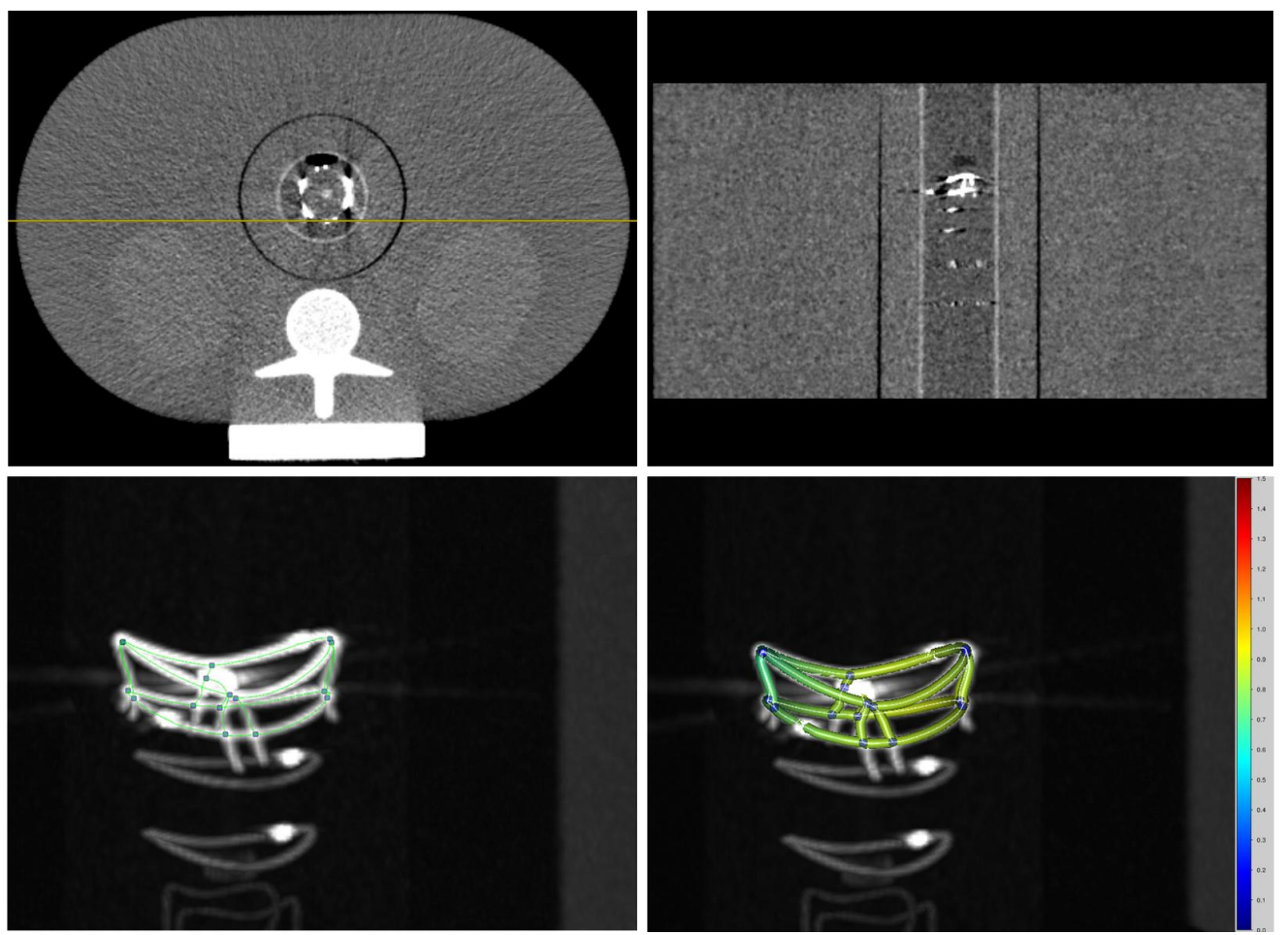

Figure 4. Example of a time-averaged volume, showing an axial (top left) and coronal (top right) multiplanar reconstruction slice and a MIP with a geometric model of the dual-ring stent (bottom). The yellow horizontal line indicates the level of the coronal slice. The green lines and blue dots in the model represent the edges and node points, respectively. The colors in the mesh represent the amplitude of the motion (bottom right) on a scale from 0 to $1.5 \mathrm{~mm}$.

quadratic polynomials to fit the $\mathrm{x}, \mathrm{y}$ and $\mathrm{z}$ subvoxel location. The dual-ring of the Anaconda has four pairs of hooks that form crossings at both the first and second ring-stent. The node points at these crossings were used to analyze the motion of the stent graft. The motion patterns as estimated by the algorithm were subtracted from the reference camera patterns to obtain absolute errors. In order to do so, the camera reference patterns were downsampled to obtain positions at 10 equidistant time-intervals that correspond to the 10 phases of the cardiac cycle in the algorithm patterns.

Table 2. Parameter settings used for image registration and segmentation.

\begin{tabular}{l|l|l|l}
\multicolumn{2}{c|}{ Registration } & \multicolumn{2}{c}{ Segmentation } \\
\hline Parameter & Value & Parameter & Value \\
\hline speed factor & 1.0 & seedThreshold & 2000 \\
scale sampling & 16 & MCPspeedfactor & $100(500)$ \\
final grid sampling & 20 & weakThreshold & 500 \\
mass transforms & $2\left(2^{\text {nd }}\right.$ order Laplacian $)$ & strongThreshold & 1800 \\
deform limit & 1.0 & expectedNumberOfEdges & 3 \\
final scale & 1.0 & & \\
grid sampling factor & 20 & &
\end{tabular}



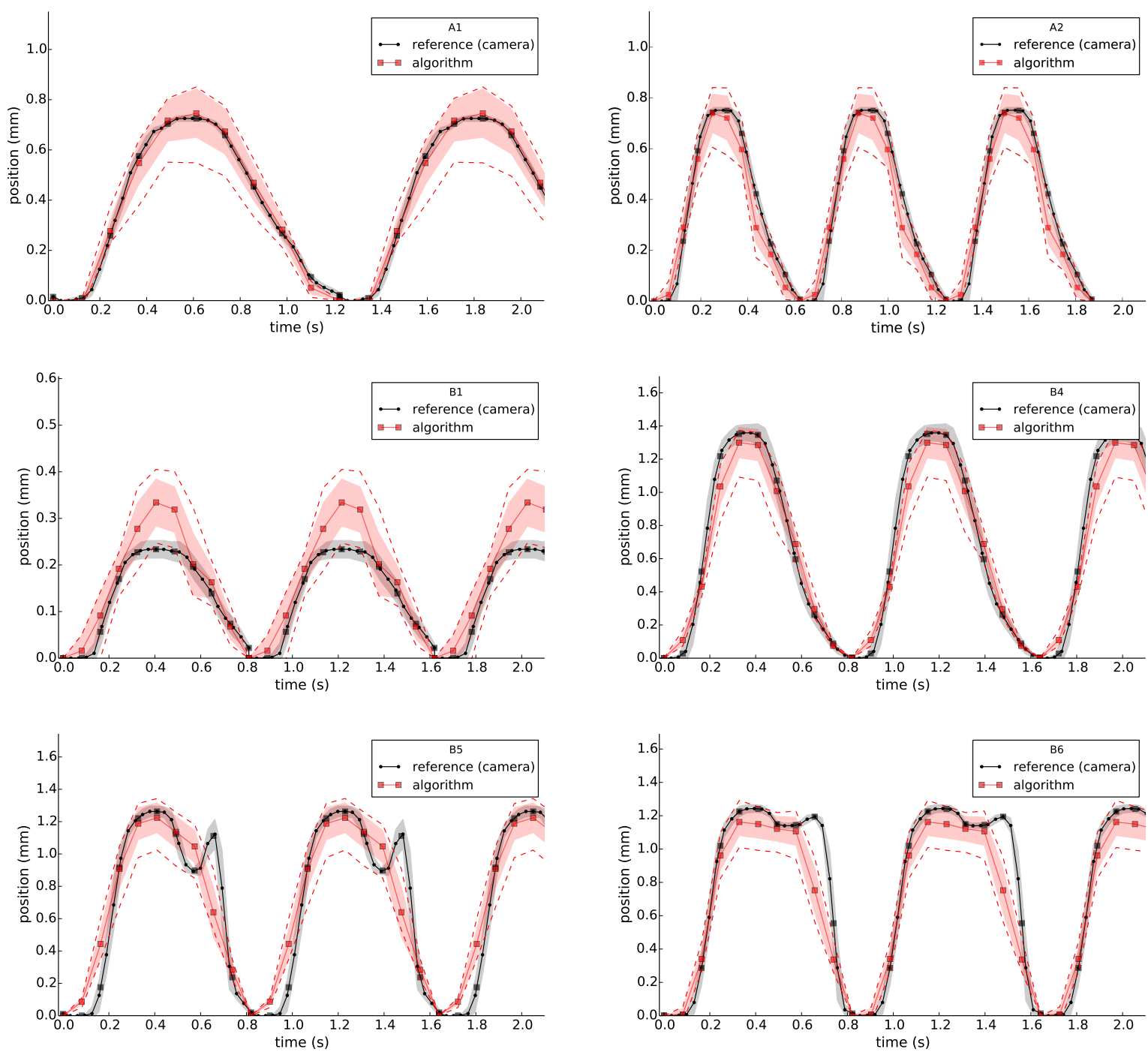

Figure 5. Displacement of six motion patterns. Solid red lines represent the mean displacement of the node points on the ring-stents as estimated by the algorithm. The standard deviation over all node points is represented by the red bounds and the dotted red lines represent the minimum and maximum positions. The reference patterns represent the mean of the recorded periods ('output simulator mean' in Figure 2). The black and red squares indicate the positions at each of the 10 phases of the cardiac cycle that were compared to obtain absolute errors.

\section{RESULTS}

An Intel Core i7-5500U processor with $2.9 \mathrm{GHz}$ clock speed and 16 GB RAM was used to perform registration, which took approximately 12 minutes for the cropped multiphase CT volumes. Parameter settings are given in Table 2 and are based on previous experimental work. ${ }^{26}$ For segmentation, the parameter values were determined experimentally for one time-averaged volume and reused for the other volumes. The parameter MCPspeedfactor determines the cost function for the MCP algorithm and required adjustment in one case (pattern B4, value between parentheses in Table 2). Figure 4 presents an example an obtained time-averaged volume and a geometric model of the dual ring-stent. An axial and coronal multi-planar reconstruction slice are shown (top) as well as a 3D maximum intensity projection (MIP) (bottom). The geometric model is shown with edges (green line) and node points (blue dots). A dynamic mesh of the model is also shown in which the colors represent the amplitude of the motion. In Figure 5 the displacement of six motion patterns is shown. The patterns as estimated by the 
algorithm are shown in red. The reference (camera) patterns are shown in black with gray bounds that show the standard deviation of the recorded periods. The difference between the red and black lines represents the error of the algorithm. The red bounds show the standard deviation of the node points on the ring-stents. The extra peak in pattern B5 and B6 was not present in the algorithm pattern. Table 3 presents the absolute amplitude errors of the patterns. The mean amplitude error was similar and small for all patterns $(<0.11 \mathrm{~mm})$. The maximum amplitude error was $0.27 \mathrm{~mm}$ (B4). The absolute errors over all 10 positions (10 phases) for all node points are presented in boxplots in Figure 6. Mean absolute errors were smaller than $0.14 \mathrm{~mm}$ in all patterns. The maximum absolute error was $0.39 \mathrm{~mm}$ (B4) and $0.63 \mathrm{~mm}$ (B6) in the patterns without and with a second peak, respectively.

Table 3. Absolute amplitude errors of the patterns. Mean, standard deviation and range over all node points are shown. Values are given in mm and as a percentage of the pattern's amplitude. Note that A3 and B3 are repeated measures with the same camera reference.

\begin{tabular}{l|l|l} 
Motion pattern & Amplitude error (mm) & Amplitude error (\%) \\
\hline A1 & $0.08 \pm 0.05(0.03-0.17)$ & $12.0 \pm 6.4(4.0-24.0)$ \\
\hline A2 & $0.07 \pm 0.04(0-0.15)$ & $9.2 \pm 5.9(1.0-19.5)$ \\
\hline A3 & $0.11 \pm 0.05(0.03-0.16)$ & $15.8 \pm 6.9(4.7-22.7)$ \\
\hline B0 & $0.04 \pm 0.03(0-0.11)$ & - \\
\hline B1 & $0.10 \pm 0.05(0.01-0.17)$ & $43.0 \pm 23.0(5.8-73.4)$ \\
\hline B2 & $0.06 \pm 0.04(0.02-0.12)$ & $14.8 \pm 9.7(4.5-31.7)$ \\
\hline B3 & $0.06 \pm 0.03(0-0.10)$ & $8.0 \pm 4.9(1.6-14.8)$ \\
\hline B4 & $0.06 \pm 0.09(0-0.27)$ & $4.7 \pm 6.9(0.3-19.7)$ \\
\hline B5 & $0.07 \pm 0.07(0-0.24)$ & $5.6 \pm 5.9(0.2-18.9)$ \\
\hline B6 & $0.09 \pm 0.08(0-0.23)$ & $6.9 \pm 6.23(0.5-18.8)$
\end{tabular}
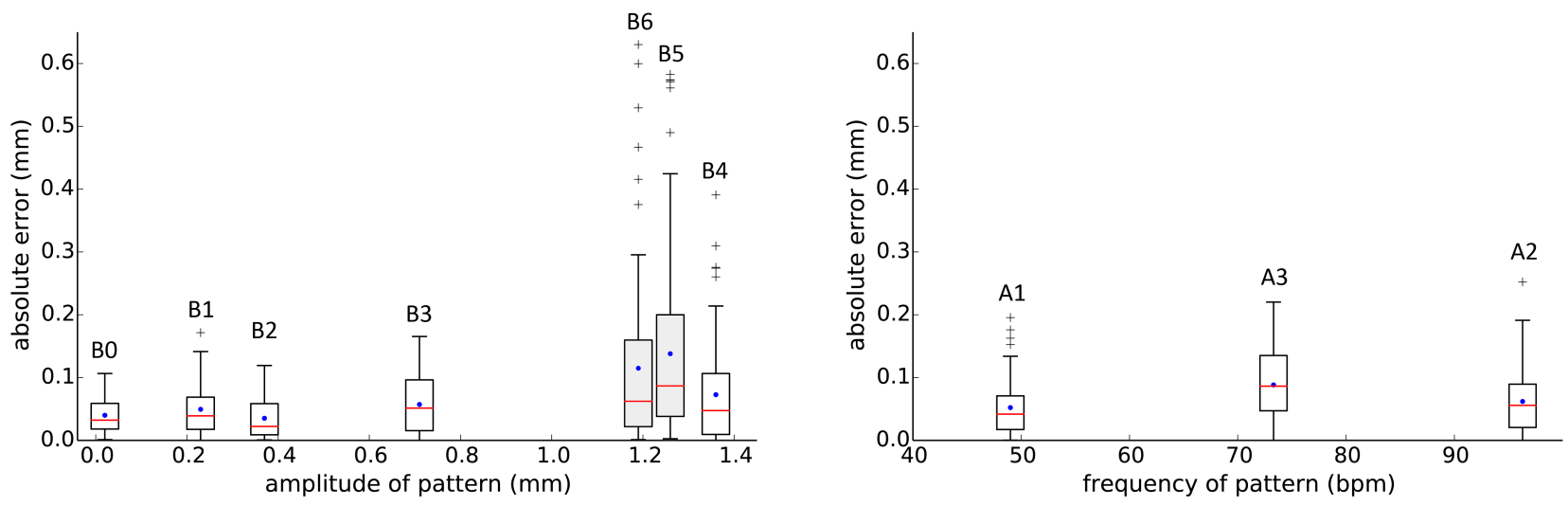

Figure 6. Absolute errors over all 10 positions (10 phases) in the displacement patterns that were estimated by the algorithm for all node points on the ring-stents. Blue dots represent the mean absolute errors. The whiskers represent 1.5 times the interquartile range. The two patterns with an extra peak (B5, B6) are shown with gray boxes.

\section{DISCUSSION}

We have evaluated the performance of our combined registration and segmentation algorithm for 10 ECG-gated CT datasets that were obtained experimentally with an Anaconda stent graft moving inside a realistic abdomen phantom. The results provide evidence that our algorithm is able to accurately measure in-vivo dynamics of aortic stent grafts on ECG-gated CT data. Pattern amplitude errors were smaller than $0.28 \mathrm{~mm}$, which is about 
half the obtained voxel size. In previous work, Klein performed experiments on synthetic $2 \mathrm{D}$ data and found the mean registration error for the Gravity algorithm well below 1 pixel. ${ }^{26}$ The current work supports this result for registration of multiphase 3D data. Although the second peaks that were added in two motion patterns (B5, B6) were not found in the algorithm patterns (Figure 5), it is expected that the gantry rotation time $(0.4 \mathrm{~s})$ of the CT scanner was the limiting factor since the width of the peaks approximates the half-rotation time. It should be further investigated whether these narrow peaks can be measured when a CT scanner with shorter rotation time is used. Furthermore, it should be taken into account that the experiments were performed in $z$-direction only. Nevertheless, it is expected that the performance in $x$ - and $y$-direction can be equal or even better considering that we obtained isotropic voxels $\left(0.5 \mathrm{~mm}^{3}\right)$ and used a slice thickness of $1 \mathrm{~mm}$. Although we simulated realistic displacement profiles for the abdominal aorta, the motion was simplified compared to the situation in vivo. In vivo, the blood flow induces motion that may deform both the stent graft and the vessel. Deformation may be more difficult to measure compared to displacement of the entire stent graft since this involves curvature changes or even twisting motion. Despite the limitations of the present work, we expect that our method can aid the prediction of failure in the individual patient, which may allow for patient specific follow-up schemes and early catheter-based reintervention before observing failure. Moreover, it can benefit preclinical testing as well as early modification of a stent graft design. Since CT is commonly routinely performed in postoperative imaging the presented method could be integrated in the clinic. Due to the increasingly fast rotation times of the scanners the radiation dose can be comparable to static protocols.

\section{CONCLUSION}

Using a physical dynamic stent graft model, we have evaluated the performance of our combined registration and segmentation algorithm to quantify stent graft motion on ECG-gated CT data. We conclude that the algorithm performs well for measuring aortic stent graft motion with amplitudes as small as $0.3 \mathrm{~mm}$. Future work will focus on the robustness of the algorithm for different registration parameters and the performance for measuring stent graft and vessel deformation due to pulsatile motion rather than displacement. Since validation of deformation is difficult in physical experimental models, the presented method will be evaluated using in silico simulations with known displacements fields or real-world patient data with extracted landmark trajectories. Our method enables further studies to the motion of implanted stent grafts and their interaction with the aortic vessel.

\section{ACKNOWLEDGMENTS}

The authors would like to thank the Department of Radiology at the Medisch Spectrum Twente for their technical support, in particular Bert Klein Rot. Furthermore, Marcel Greuter and the department of Radiology at the University Medical Center Groningen for providing us their motion simulator to perform test measurements. The work was funded by an unrestricted research grant from Vascutek, a TERUMO Company.

\section{REFERENCES}

[1] Buck, D. B., van Herwaarden, J. A., Schermerhorn, M. L., and Moll, F. L., "Endovascular treatment of abdominal aortic aneurysms.," Nat. Rev. Cardiol. 11(2), 112-23 (2014).

[2] Hobo, R. and Buth, J., "Secondary interventions following endovascular abdominal aortic aneurysm repair using current endografts. A EUROSTAR report," J. Vasc. Surg. 43(5), 896-902 (2006).

[3] England, A., García-Fiñana, M., Fisher, R. K., Naik, J. B., Vallabhaneni, S. R., Brennan, J. A., and McWilliams, R. G., "Migration of fenestrated aortic stent grafts," J. Vasc. Surg. 57(6), 1543-1552 (2013).

[4] Troisi, N., Donas, K. P., Austermann, M., Tessarek, J., Umscheid, T., and Torsello, G., "Secondary procedures after aortic aneurysm repair with fenestrated and branched endografts.," J. Endovasc. Ther. 18(2), 146-53 (2011).

[5] Verhoeven, E. L. G., Vourliotakis, G., Bos, W. T. G. J., Tielliu, I. F. J., Zeebregts, C. J., Prins, T. R., Bracale, U. M., and van den Dungen, J. J. A. M., "Fenestrated Stent Grafting for Short-necked and Juxtarenal Abdominal Aortic Aneurysm: An 8-Year Single-centre Experience," Eur. J. Vasc. Endovasc. Surg. 39(5), 529-536 (2010). 
[6] De Bruin, J. L., Baas, A. F., Buth, J., Prinssen, M., Verhoeven, E. L. G., Cuypers, P. W. M., van Sambeek, M. R. H. M., Balm, R., Grobbee, D. E., and Blankensteijn, J. D., "Long-term outcome of open or endovascular repair of abdominal aortic aneurysm.," N. Engl. J. Med. 362(20), 1881-1889 (2010).

[7] Harris, P. L., Vallabhaneni, S. R., Desgranges, P., Becquemin, J. P., Van Marrewijk, C., and Laheij, R. J. F., "Incidence and risk factors of late rupture, conversion, and death after endovascular repair of infrarenal aortic aneurysms: The EUROSTAR experience," J. Vasc. Surg. 32(4), 739-749 (2000).

[8] Roos, J. E., Hellinger, J. C., Hallet, R., Fleischmann, D., Zarins, C. K., and Rubin, G. D., "Detection of endograft fractures with multidetector row computed tomography," J. Vasc. Surg. 42(5), 1002-1006 (2005).

[9] Klein, A., Oostveen, L. J., Greuter, M. J. W., Hoogeveen, Y., Schultze Kool, L. J., Slump, C. H., and Renema, W. K. J., "Detectability of Motions in AAA With ECG-gated CTA: a Quantitative Study," Med. Phys. 36(10), 4616-4624 (2009).

[10] Klein, A., Renema, W., Vliet, J. A., Oostveen, L. J., Hoogeveen, Y., Schultze Kool, L. J., and Slump, C. H., [Diagnosis, Screening and Treatment of Abdominal, Thoracoabdominal and Thoracic Aortic Aneurysms], InTech, Rijeka, 125-144 (2011).

[11] Langs, G., Paragios, N., Desgranges, P., Rahmouni, A., and Kobeiter, H., "Learning deformation and structure simultaneously: In situ endograft deformation analysis," Med. Image Anal. 15(1), 12-21 (2011).

[12] Langs, G., Paragios, N., Donner, R., Desgranges, P., Rahmouni, A., and Kobeiter, H., "Motion analysis of endovascular Stent-Grafts by MDL based registration," Proc. IEEE 11th ICCV, 1-8 (2007).

[13] Teutelink, A., Rutten, A., Muhs, B. E., Olree, M., van Herwaarden, J. A., de Vos, A. M., Prokop, M., Moll, F. L., and Verhagen, H. J. M., "Pilot Study of Dynamic Cine CT Angiography for the Evaluation of Abdominal Aortic Aneurysms: Implications for Endograft Treatment," J. Endovasc. Ther. 13(2), 139-144 (2006).

[14] Teutelink, A., Muhs, B. E., Vincken, K. L., Bartels, L. W., Cornelissen, S. A., van Herwaarden, J. A., Prokop, M., Moll, F. L., and Verhagen, H. J. M., "Use of dynamic computed tomography to evaluate preand postoperative aortic changes in AAA patients undergoing endovascular aneurysm repair.," J. Endovasc. Ther. 14(1), 44-49 (2007).

[15] Iezzi, R., Di Stasi, C., Dattesi, R., Pirro, F., Nestola, M., Cina, A., Codispoti, F. A., Snider, F., and Bonomo, L., "Proximal aneurysmal neck: dynamic ECG-gated CT angiography-conformational pulsatile changes with possible consequences for endograft sizing.," Radiology 260(2), 591-598 (2011).

[16] Van Keulen, J. W., Moll, F. L., Barwegen, G. K., Vonken, E. P. A., and Van Herwaarden, J. A., "Pulsatile distension of the proximal aneurysm neck is larger in patients with stent graft migration," Eur. J. Vasc. Endovasc. Surg. 40(3), 326-331 (2010).

[17] Ganten, M. K., Krautter, U., Von Tengg-Kobligk, H., Böckler, D., Schumacher, H., Stiller, W., Delorme, S., Kauczor, H. U., Kauffmann, G. W., and Bock, M., "Quantification of aortic distensibility in abdominal aortic aneurysm using ECG-gated multi-detector computed tomography," Eur. Radiol. 18(5), 966-973 (2008).

[18] van Keulen, J. W., Vincken, K. L., van Prehn, J., Tolenaar, J. L., Bartels, L. W., Viergever, M. A., Moll, F. L., and van Herwaarden, J. A., "The Influence of Different Types of Stent Grafts on Aneurysm Neck Dynamics after Endovascular Aneurysm Repair," Eur. J. Vasc. Endovasc. Surg. 39(2), 193-199 (2010).

[19] Pol, J. A., Truijers, M., van der Vliet, J. A., Fillinger, M. F., Marra, S. P., Renema, W. K. J., Oostveen, L. J., Kool, L. J. S., and Blankensteijn, J. D., "Impact of dynamic computed tomographic angiography on endograft sizing for endovascular aneurysm repair," J. Endovasc. Ther. 16(5), 546-551 (2009).

[20] Muhs, B. E., Vincken, K. L., van Prehn, J., Stone, M. K. C., Bartels, L. W., Prokop, M., Moll, F. L., and Verhagen, H. J. M., "Dynamic Cine-CT Angiography for the Evaluation of the Thoracic Aorta; Insight in Dynamic Changes with Implications for Thoracic Endograft Treatment," Eur. J. Vasc. Endovasc. Surg. 32(5), 532-536 (2006).

[21] Wentz, R., Manduca, A., Fletcher, J. G., Siddiki, H., Shields, R. C., Vrtiska, T., Spencer, G., Primak, A. N., Zhang, J., Nielson, T., McCollough, C., and Yu, L., "Automatic segmentation and co-registration of gated CT angiography datasets: measuring abdominal aortic pulsatility," Proc. SPIE 6511, 65111I-65111I-9 (2007).

[22] Zandvoort, H. J. A., Moll, F. L., Domanian, A., Van Keulen, J. W., Vonken, E. J. P. A., and Van Herwaarden, J. A., "Preoperative infra- and suprarenal aortic pulsatile distension is comparable between relatively young and older patients with an abdominal aortic aneurysm," Ann. Vasc. Surg. 28(4), 845-849 (2014). 
[23] van Herwaarden, J. A., Bartels, L. W., Muhs, B. E., Vincken, K. L., Lindeboom, M. Y. A., Teutelink, A., Moll, F. L., and Verhagen, H. J. M., "Dynamic magnetic resonance angiography of the aneurysm neck: Conformational changes during the cardiac cycle with possible consequences for endograft sizing and future design," J. Vasc. Surg. 44(1), 22-28 (2006).

[24] van Prehn, J., van Herwaarden, J. A., Vincken, K. L., Verhagen, H. J. M., Moll, F. L., and Bartels, L. W., "Asymmetric aortic expansion of the aneurysm neck: Analysis and visualization of shape changes with electrocardiogram-gated magnetic resonance imaging," J. Vasc. Surg. 49(6), 1395-1402 (2009).

[25] van Herwaarden, J. A., Muhs, B. E., Vincken, K. L., van Prehn, J., Teutelink, A., Bartels, L. W., Moll, F. L., and Verhagen, H. J. M., "Aortic Compliance Following EVAR and the Influence of Different Endografts: Determination Using Dynamic MRA," J. Endovasc. Ther. 13(3), 406-414 (2006).

[26] Klein, A., [Segmentation and motion estimation of stent grafts in abdominal aortic aneurysms], Gildeprint B.V., Enschede, 121-142 (2011).

[27] QRM (Quality assurance in Radiology and Medicine), "Motion Simulator Sim2D." 2008 http://www .qrm. de/content/products/dynamic/sim2d.htm. (Accessed: 20 May 2015).

[28] Hazer, D., Finol, E. A., Kostrzewa, M., Kopaigorenko, M., Richter, G., and Dillmann, R., "Computational biomechanics and experimental validation of vessel deformation based on $4 \mathrm{D}-\mathrm{CT}$ imaging of the porcine aorta," Proc. SPIE 7262, 72621F (2009).

[29] Flora, H. S., Woodhouse, N., Robson, S., and Adiseshiah, M., "Micromovements at the aortic aneurysm neck measured during open surgery with close-range photogrammetry: Implications for aortic endografts," J. Endovasc. Ther. 8(5), 511-520 (2001).

[30] Arko, F. R., Murphy, E. H., Davis, C. M., Johnson, E. D., Smith, S. T., and Zarins, C. K., "Dynamic geometry and wall thickness of the aortic neck of abdominal aortic aneurysms with intravascular ultrasonography," J. Vasc. Surg. 46(5), 891-897 (2007).

[31] van Keulen, J. W., van Prehn, J., Prokop, M., Moll, F. L., and van Herwaarden, J. A., "Dynamics of the Aorta Before and After Endovascular Aneurysm Repair: A Systematic Review," Eur. J. Vasc. Endovasc. Surg. 38(5), 586-596 (2009).

[32] Klein, A., van der Vliet, J. A., Oostveen, L. J., Hoogeveen, Y., Schultze Kool, L. J., Renema, W. K. J., and Slump, C. H., "Automatic segmentation of the wire frame of stent grafts from CT data," Med. Image Anal. 16(1), 127-139 (2012).

[33] Klein, A., Kroon, D.-J., Hoogeveen, Y., Schultze Kool, L. J., Renema, W. K. J., and Slump, C. H., "Multimodal image registration by edge attraction and regularization using a B-spline grid," Proc. SPIE 7962, 796220-796220-8 (2011).

[34] Klein, A., "Python Image Registration Toolkit (PIRT)." Available at: https://bitbucket.org/ almarklein/pirt (2014). 